\title{
Tumor fibroso solitario gigante de pleura
}

\author{
Javier Pérez Palenzuela ${ }^{1}$ y Kymani Pérez García ${ }^{1}$
}

"Hospital Clínico Quirúrgico "Hermanos Ameijeiras". La Habana, Cuba.

Recibido 2021-03-29, aceptado 2021-04-23

Correspondencia a: Dr. Javier Pérez Palenzuela jape122@gmail.com

\section{Giant solitary fibrous tumor of the Pleura}

Aim: To report the case of a $19 \mathrm{~cm}$ diameter giant mass in the left hemithorax in a 59-year-old patient who presented with dyspnea, cough and chest pain, confirmed by imaging study. Materials and Method: Clinical record of a patient who was diagnosed with a solitary fibrous tumor of the pleura, undergoing surgery to excise the lesion. Results: A left posterolateral thoracotomy was performed to excise the giant tumor, also requiring resection of the diaphragm and partial pericardiectomy with favorable evolution. Discussion: The solitary fibrous tumor is a rare neoplasm derived from the mesenchyme that most commonly affects the pleura, typically well circumscribed, pedunculated, with vessels within the tumor pedicle, and can become large, being considered giant when they are larger than $15 \mathrm{~cm}$ diameter. Conclusion: The correct diagnosis is of vital importance, since surgical resection it a potentially curable treatment. Surgical treatment can be performed by thoracotomy or videothoracoscopy, depending on the size of the tumor. Despite the benign behavior, it requires long-term follow-up due to the tendency to recur.

Keywords: solitary fibrous tumor; giant pleural tumor; surgical treatment.

\section{Resumen}

Objetivo: Reportar el caso de una masa gigante en hemitórax izquierdo de $19 \mathrm{~cm}$ de diámetro en un paciente de 59 años que debutó con disnea, tos y dolor torácico, confirmándose por estudio imagenológico. Materiales y Método: Registro clínico de un paciente al cual se le diagnostica tumor fibroso solitario de pleura, siendo intervenido quirúrgicamente para exéresis de la lesión. Resultados: Se realiza toracotomía posterolateral izquierda para exéresis de tumor gigante, requiriendo además, resección de diafragma y pericardiectomía parcial con evolución favorable. Discusión: El tumor fibroso solitario es una neoplasia rara derivada del mesénquima que afecta más comúnmente a la pleura, típicamente bien circunscrita, pediculada, con vasos dentro del pedículo tumoral, pudiendo llegar a ser de gran tamaño, siendo considerados gigantes cuando tienen más de $15 \mathrm{~cm}$ de diámetro. Conclusión: El diagnóstico correcto es de vital importancia, ya que con la resección quirúrgica es potencialmente curable. El tratamiento quirúrgico puede efectuarse por toracotomía o videotoracoscopia, dependiendo del tamaño del tumor. A pesar del comportamiento benigno, requiere seguimiento a largo plazo debido a la tendencia a la recidiva.

Palabras clave: tumor fibroso solitario; tumor pleural gigante; tratamiento quirúrgico.

\section{Introducción}

El tumor fibroso solitario (TFS) es una neoplasia rara que afecta más comúnmente a la pleura, aunque ha sido descrito en otras localizaciones extrapleurales como el mediastino, pericardio, mesenterio, peritoneo, espacios extraperitoneales, nariz y senos paranasales ${ }^{1}$.

El primer caso de TFS fue descrito histológicamente por Wagner en 1870, sin embargo, el primer tumor pleural fue presentado por Lieutaud en 1767. En 1931, Klemperer y Rabin publicaron una des- cripción histopatológica y dividieron estos tumores en 2 categorías: difuso y localizado. Stout y Murray en 1942 identificaron el origen mesenquimatoso de este tipo de tumores, demostrado posteriormente por la microscopía electrónica e inmunohistoquímica. A lo largo del tiempo estos tumores han recibido varias denominaciones: mesotelioma localizado, mesotelioma benigno, mesotelioma fibroso, fibroma pleural, fibromixoma pleural y tumor fibroso localizado ${ }^{2}$.

Se describe su origen en fibroblastos derivados del mesénquima submesotelial y se caracteriza por 
la formación de tumores localizados, generalmente únicos, que no producen derrame pleural ${ }^{3}$.

Las características por imagen del TFS pleural son su presentación como masa extrapulmonar o cisural, bien definida y con componentes heterogéneos en relación con áreas de necrosis y hemorragia.

El tratamiento es quirúrgico y su pronóstico es favorable en la mayoría de los casos, sin embargo, la recurrencia del mismo indica degeneración maligna y el pronóstico es pobre.

Teniendo en cuenta que en la literatura se han descrito aproximadamente entre 900 y 1.760 casos de esta neoplasia, la cual representa cerca del $8 \%$ de todos los tumores benignos intratorácicos y el 5\% de los tumores pleurales ${ }^{4}$, nos motivamos a describir su diagnóstico y tratamiento en un paciente de 59 años intervenido quirúrgicamente en nuestro servicio.

\section{Descripción de caso}

Paciente masculino de 59 años que 3 meses antes del ingreso comienza con astenia, disnea leve a los esfuerzos, tos seca esporádica y dolor en región inferior de hemitórax izquierdo que fue incrementándose en intensidad, requiriendo tratamiento analgésico periódico. Asociado a esto presentó episodios esporádicos siempre nocturnos de fatiga y sudoración profusa que cedían con la ingestión de bebidas o comidas azucaradas, sin lograrse constatar cifras de glicemia. Es llevado a un centro hospitalario donde le realiza radiografía de tórax y se reportó gran radiopacidad en tercio inferior del hemitórax izquierdo, que provocaba desplazamiento de estructuras mediastinales hacia el lado contralateral. Por este motivo se decide realizar tomografía axial computarizada con contraste endovenoso que informa gran masa tumoral en región paracardíaca izquierda, impresionando extrapulmonar, heterogénea, con áreas de necrosis, con compresión del pulmón izquierdo. Dicha masa se encuentra rodeada de grasa y mantiene su interfase con el área cardiaca midiendo $18 \mathrm{~cm}$ x $12 \mathrm{~cm}$ x $11 \mathrm{~cm}$. Ligero derrame pleural izquierdo sin definirse lesiones en el parénquima pulmonar. No alteraciones abdominales (Figura 1).

El ecocardiograma informó buena contractilidad global y segmentaria. Sin crecimiento de cavidades, ni engrosamiento miocárdico, ni derrame pericárdi-

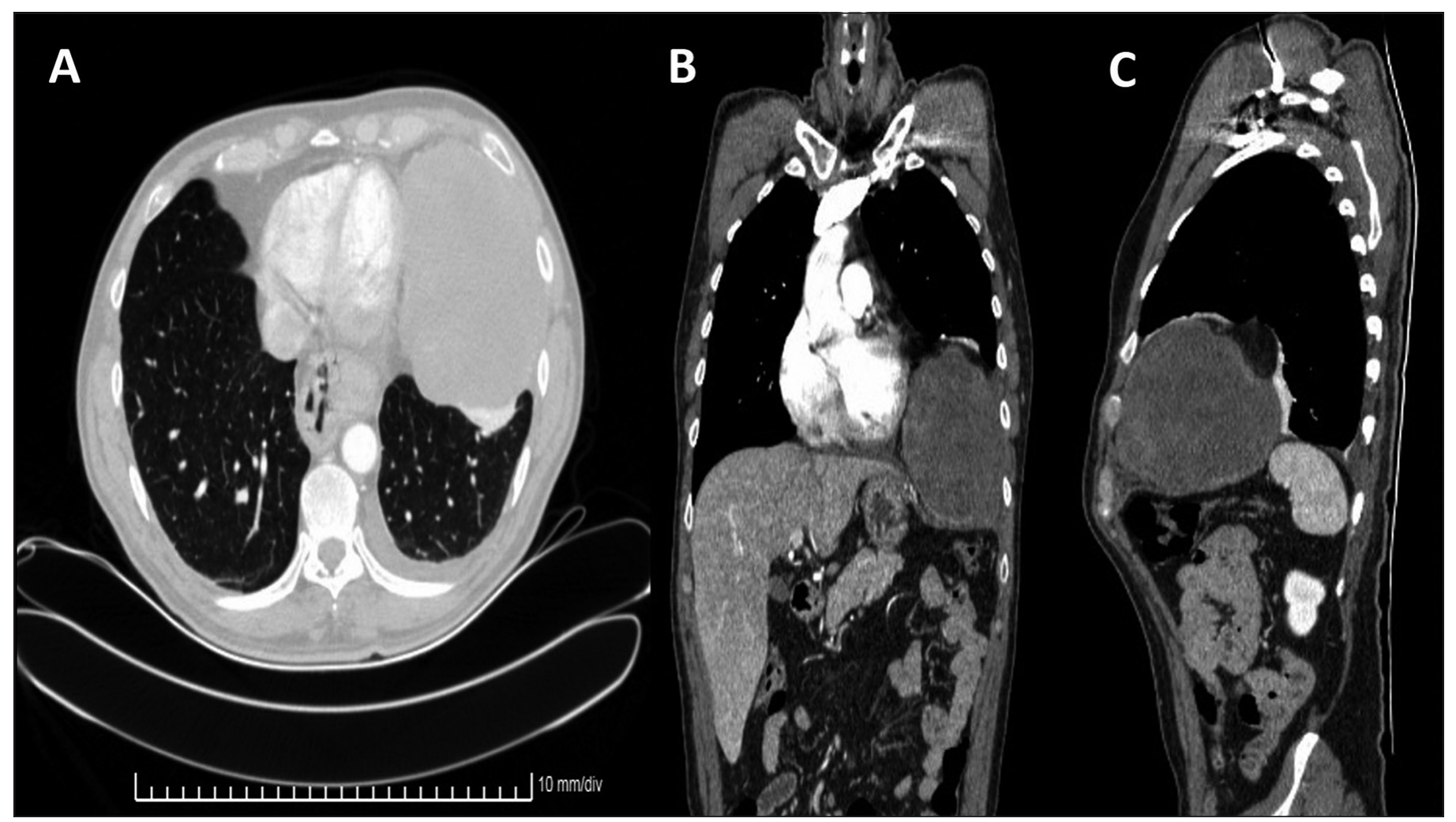

Figura 1. A: vista axial, B: vista coronal y C: vista sagital e la TC de tórax donde se aprecia gran masa que desplaza diafragma y corazón. 


\section{CASOS CLÍNICOS}

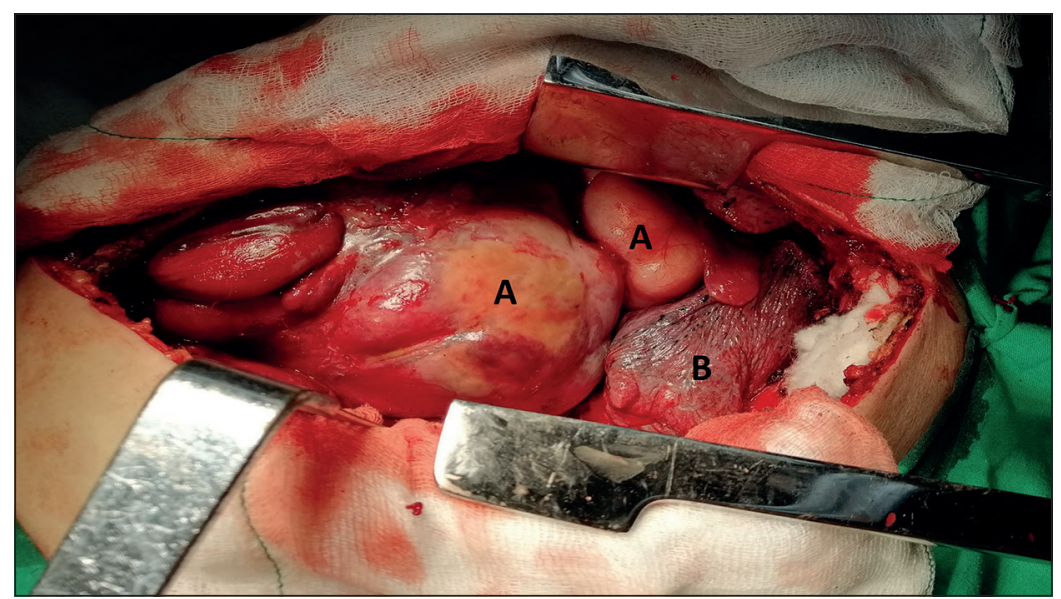

Figura 2. Apertura del tórax apreciándose: A: tumor polilobulado firmemente adherida a diafragma y B: pulmón colapsado sin adherencias a la masa.

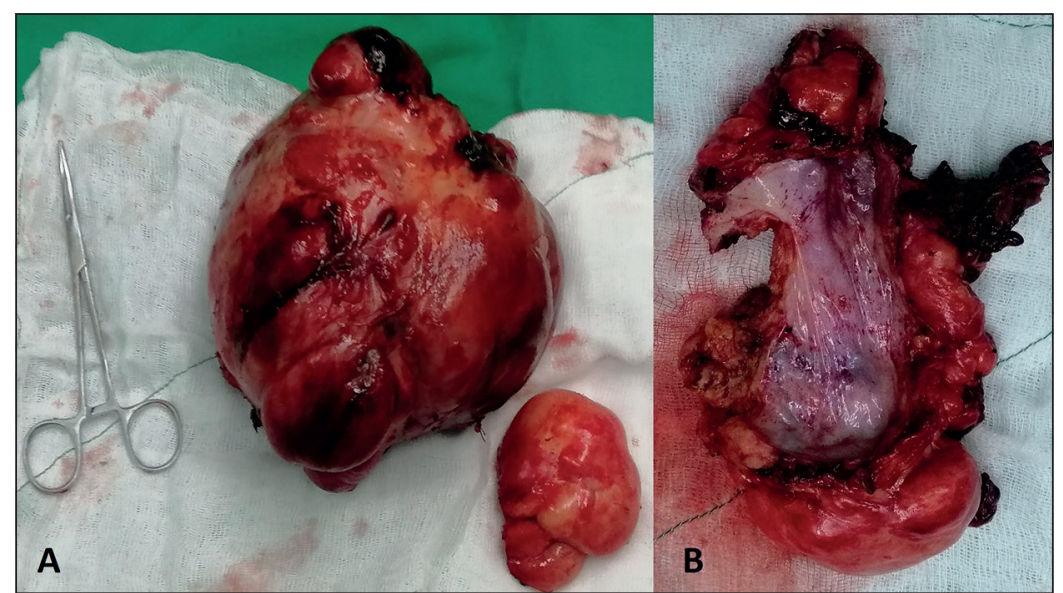

Figura 3. A: tumor extirpado en su totalidad y B: fragmento de pericardio resecado.

Figura 4. A: diafragma resecado parcialmente con estomago protruyendo a través del mismo. B: corazón expuesto después de la pericardiectomía parcial y C: pulmón reexpandido. co, ni trombos. La masa pleural desplaza, además, al corazón hacia la línea media y hacia abajo.

Se le realiza biopsia por tru-cut que informa TFS pleural con inmunohistoquímica que reporta $\mathrm{CK}$ negativo, Vimentina positivo, S-100 negativo, Alfactina negativa, WT1 positivo, Calretinina negativa y KI-67 positivo en un 5\%.

Con este diagnóstico es trasladado a nuestro servicio de cirugía donde se decide intervenir quirúrgicamente. Es llevado al salón de operaciones y se realiza toracotomía posterolateral izquierda con abordaje del tórax a nivel del $6 .^{\circ}$ espacio intercostal. Se encuentra gran tumoración polilobulada, adherida firmemente a zona medial de hemidiafragma y a pericardio. Pulmón completamente libre (Figura 2).

Para lograr la exposición del tumor se reseca 7. ${ }^{a}$ costilla, con resección parcial de diafragma y pericardiectomía por engrosamiento de la pleura que tapiza el pericardio (Figuras 3 y 4). El defecto diafragmático al no ser muy extenso se reparó con sutura primaria. Un plano interno con puntos discontinuos de colchonero con Prolene 1-0 y un plano externo con sutura continua de Prolene 2-0. El defecto pericárdico no fue reparado (Figura 5).

La evolución posoperatoria fue favorable, sin disnea, dolor torácico de moderada intensidad y sin

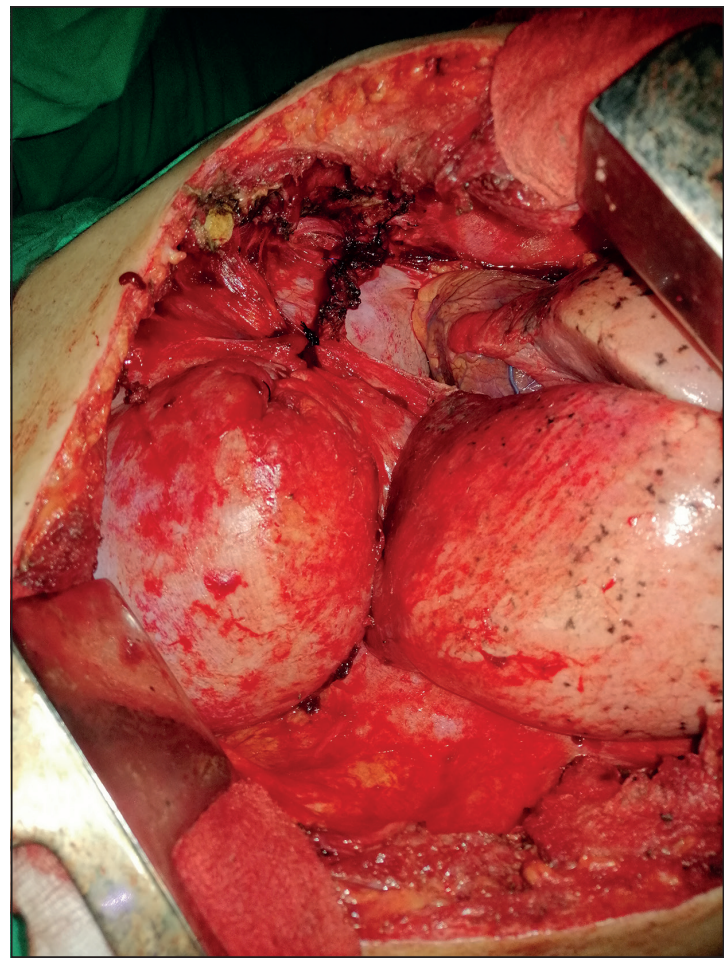

Figura 5. Diafragma reparado después de la resección. 


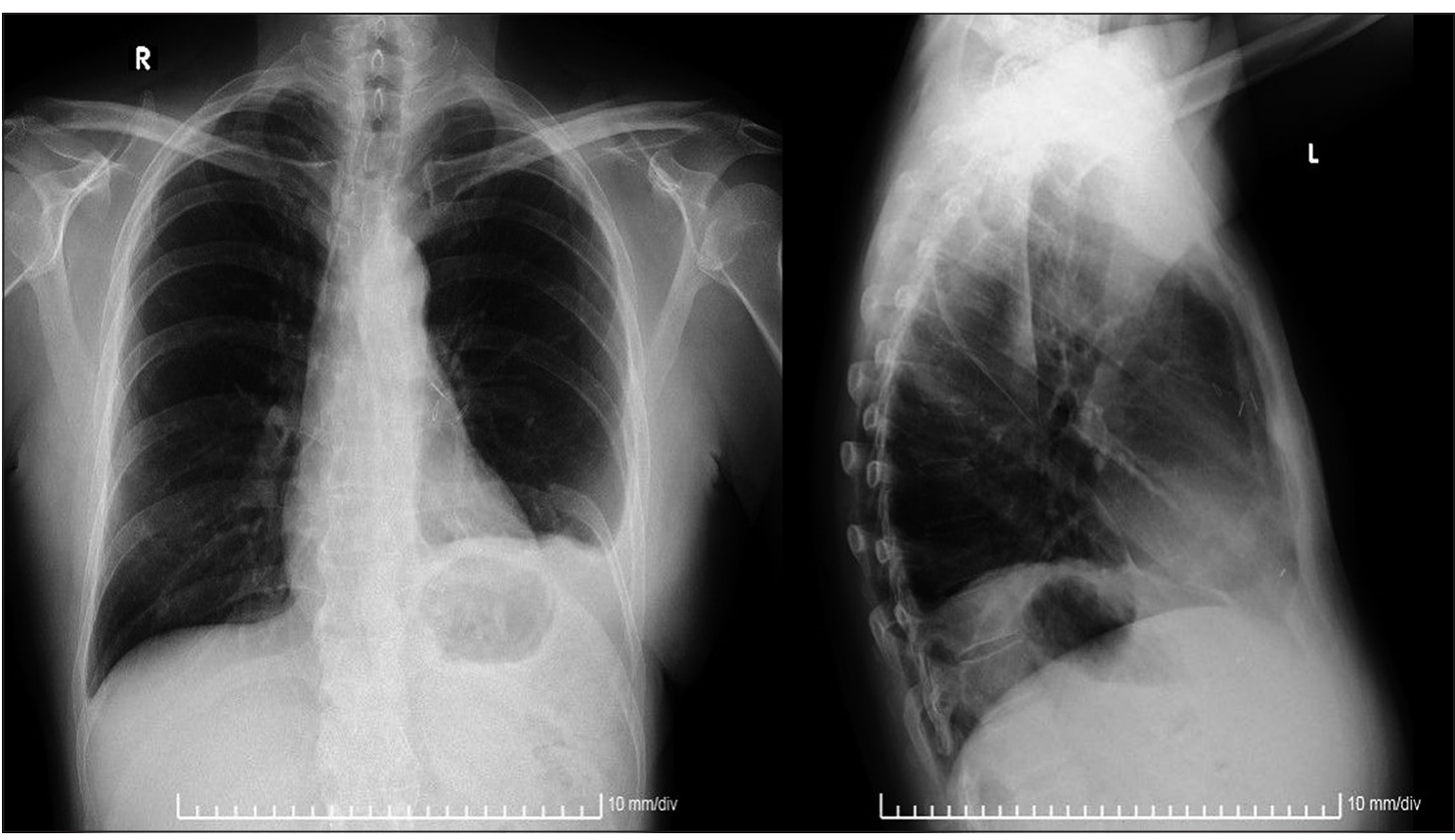

Figura 6. Radiografía de tórax de seguimiento posoperatorio. fuga gaseosa, lo que permitió el alta $72 \mathrm{~h}$ después con seguimiento por consulta, presentando hasta la fecha evolución favorable con remisión de los síntomas preoperatorios (Figura 6).

El estudio histológico del espécimen quirúrgico informó exéresis completa de TFS benigno pleural de $19 \times 11 \times 14 \mathrm{~cm}$, con índice de mitosis inferior a 4 por 10 campos, un Ki-67 de 5\% y algunas áreas de necrosis. Los márgenes de sección diafragmáticos se encontraban negativos a $1,5 \mathrm{~cm}$ del pedículo tumoral. El pericardio resecado, aunque macroscópicamente impresionaba engrosado presentó características histológicas normales.

\section{Discusión}

EL TFS es una neoplasia rara, con incidencia de alrededor de 2,8 casos por 100.000 pacientes registrados al año ${ }^{5}$. En Latinoamérica existen pocas series de casos de esta enfermedad ${ }^{6}$. Puede ser encontrados en un rango de edad de 5 a 87 años, con una frecuencia mayor hacia la 5. ${ }^{\mathrm{a}}-6 .{ }^{\mathrm{a}}$ década de la vida y con ligero predominio en el sexo femenino ${ }^{7}$.

Mientras que los TFS benignos son usualmente pequeños y pediculados, la mayoría de los TFS malignos pueden alcanzar los $10 \mathrm{~cm}$ y más. Sin embargo, los benignos son considerados gigantes cuando su diámetro es mayor de $15 \mathrm{~cm}$ o cuando el tumor ocupa más del $40 \%$ del hemitórax ${ }^{8}$. Se plantea que $2 / 3$ de los TFS se desarrollan en la pleura visceral, mientras que $1 / 3$ lo hace de la pleura parietal, donde el tumor es con frecuencia más grande y adherencias más amplias?.

La mayoría de los pacientes con TFS son asintomáticos al momento de ser diagnosticados incidentalmente por una radiografía de tórax de rutina. Las lesiones grandes pueden provocar compresión de estructuras vecinas y el paciente puede presentar algunos síntomas tales como dolor torácico, tos $\mathrm{y}$ disnea ${ }^{10}$. Algunos pacientes debutan con los llamados síndromes paraneoplásicos que incluyen hipoglicemia refractaria y osteoartropatía pulmonar hipertrófica, los cuales mejoran inmediatamente tras la resección del tumor ${ }^{11}$.

La TC de tórax es una herramienta muy útil en el diagnóstico, permite claramente identificar la localización y el tamaño de la lesión, ayudando a los cirujanos a definir la posibilidad de resección. Generalmente este estudio muestra una masa homogénea y bien circunscrita, sin embargo, en tumores grandes o gigantes como el presentado en nuestro paciente, generalmente se describen con densidad heterogénea debido a la hemorragia intratumoral, necrosis y cambios quísticos ${ }^{12}$.

Desde el punto de vista microscópico las mitosis 
son raramente visibles en TFS benignos siendo menores de 3 mitosis por 10 campos de alto poder. El estudio inmunohistoquímico por su parte va a ayudar a excluir carcinoma sarcomatoide periférico de pulmón, mesotelioma maligno, sarcoma sinovial, histiocitoma fibroso maligno entre otros tumores $\operatorname{raros}^{3}$.

La resección quirúrgica completa que puede llegar a resección pulmonar si el origen es la pleura visceral es el proceder de elección en todos los TFS. Generalmente, si el tumor es pequeño, una exéresis adecuada puede ser lograda mediante cirugía torácica video asistida, siendo la mayoría de estos benignos $^{13}$. Sin embargo, cuando los tumores son inusualmente grandes o gigantes como el descrito en nuestro paciente, será necesaria una toracotomía para remover completamente la lesión ${ }^{14}$. Algunos autores recomiendan la embolización preoperatoria de las arterias que suministran el aporte de sanguíneo a un TFS gigante debido al riesgo de hemorragia $^{8}$. El tumor en el presente caso se encontraba firmemente adherido al diafragma por un grueso pedículo mediante cual provenía su principal irrigación, sin embargo, la embolización no se realizó porque la masa no mostraba manifestaciones invasivas y se consideró que el pedículo podía ser fácilmente tratado. Como consecuencia de esto la operación fue más difícil de lo esperado, debido al gran tamaño tumoral y la exposición deficiente, lo que generó un sangrado intraoperatorio de $1.000 \mathrm{ml}$.

A pesar de que los TFS benignos pueden casi siempre ser curados con la exéresis quirúrgica completa, el seguimiento a largo plazo es necesario debido a la posibilidad de recurrencia local ${ }^{15}$. Se ha comprobado que del total de casos que desarrollan recurrencia el $23 \%$ de esta se produce después de los 5 años ${ }^{16}$.

\section{Conclusiones}

El caso reportado describe un raro tumor fibroso solitario gigante de pleura que fue satisfactoriamente resecado quirúrgicamente. Para lograr esto, la tomografía axial computarizada de tórax es un método diagnóstico de imagen fundamental, demostrando las características y el patrón tumoral. Sin embargo, el diagnóstico confirmatorio dependerá de estudios histológicos e inmunohistoquímicos. La resección completa es un método efectivo de tratamiento de este tipo de neoplasia, pero un seguimiento a largo plazo será necesario para una detección temprana de recurrencia tumoral.

\section{Responsabilidades éticas}

Protección de personas y animales. Los autores declaran que para esta investigación no se han realizado experimentos en seres humanos ni en animales.

Confidencialidad de los datos. Los autores declaran que en este artículo no aparecen datos de pacientes.

Conflictos de interés: no hay.

El artículo ha sido aprobado para su publicación por el Comité de ética local de nuestra institución.

Contribuciones de los autores: Dr. Javier Pérez Palenzuela. Cirujano del caso. Redacción, edición de imágenes, búsqueda de bibliografía, publicación en la plataforma de la revista. Dr. Kymani Pérez García. Cirujano del caso. Revisión, corrección y aprobación final del manuscrito del mismo.

\section{Bibliografía}

1. Rosado-de-Christenson ML, Abbott GF, McAdams HP, Franks TJ, Galvin JR. From the archives of the AFIP: Localized fibrous tumor of the pleura. Radiographics 2003;23:759-83 https://doi.org/10.1148/ rg. 233025165 .

2. Savu C, Melinte A, Posea R, Galie N, Balescu I, Diaconu C, et al. Pleural solitary fibrous tumors-A retrospective study on 45 patients. Medicina (Kaunas) 2020;56:185. http://dx.doi.org/10.3390/ medicina56040185.

3. Mitchel JD. Solitary fibrous tumor of the pleura. Semin Thorac Cardiovasc Surg. 2003;15:305-9. https://doi.org/10.1016/ s1043-0679(03)70011-2.

4. Erb CT, Johnson KM, Kim AW. Rare pleural tumors. Clin Chest Med. 2013;34:113-36. https://doi.org/10.1016/j. ccm.2012.12.001

5. You YH, Liu RT, Zhang Y. A large solitary fibrous tumour of the pleura: a case report and review of the literature. J Int Med Res. 2018;46:1672-7. http://dx.doi. org/10.1177/0300060517750534.

6. Santolaya R, Meneses C, López J, Prats R, Fica M, González C, et al. Tumor fibroso solitario de la pleura. Análisis de 41 casos. Rev Chil Enf Respir. 2007; 23:11-6. http://dx.doi.org/10.4067/S071773482007000100002.

7. Ershadi R, Rahim M, Abbasi M, Erfanian R. Giant solitary fibrous tumor of the pleura. J Surg Case Rep. 2018;2018:rjy270. https://doi.org/10.1093/ jscr/rjy270.

8. Perrotta F, Cerqua F, Cammarata A, Izzo A, Bergaminelli C, Curcio C, et al. Integrated therapeutic approach to giant solitary fibrous tumor of the pleura: Report of a case and review of the literature. Open Med. (Wars) 2016;11:2205. https://doi.org/10.1515/med-2016-0042. 
9. Bukamur H, Karem E, Fares S, Sigdel S, Alkhankan E, Zeid F. A series of two cases of rare tumors: Solitary fibrous tumor of the pleura. Respir Med Case Rep. 2019;28:100872. https://doi.org/10.1016/j. rmcr.2019.100872.

10. Tan JH, Hsu AA. Challenges in diagnosis and management of giant solitary fibrous tumour of pleura: a case report. BMC Pulm Med. 2016;16:114 https://doi.org/10.1186/s12890-016-02790 .

11. Karki A, Yang J, Chauhan S. Paraneoplastic syndrome associate with solitary fibrous tumor of pleura. Lung India 2018;35:245-7. http://dx.doi. org/10.4103/lungindia.lungindia_118_17.
12. Gupta A, Souza CA, Sekhon HS, Gomes MM, Hare SS, Agarwal PP, et al. Solitary fibrous tumour of pleura: CT differentiation of benign and malignant types. Clin Radiol. 2017;72:796. e9-796.e17. https://doi.org/10.1016/j. crad.2017.03.028.

13. Masuom SH, Bagheri R, Sadrizadeh A, Dalouee MN, Taherian A, Rajaie Z. Outcome of surgery in patients with solitary fibrous tumors of the pleura. Asian Cardiovasc Thorac Ann. 2016;24:18-22. https://doi. org/10.1177/0218492315614977.

14. Yanagiya M, Matsumoto J, Miura T, Horiuchi H. Extended thoracotomy with subcostal incision for giant solitary fibrous tumor of the diaphragm. AME Case Rep. 2017;1:8. http://dx.doi.org/10.21037/ acr.2017.11.02

15. Yao MJ, Ding L, Atay SM, Toubat O, Ebner P, David EA, et al. A modern reaffirmation of surgery as the optimal treatment for solitary fibrous tumors of the pleura. Ann Thorac Surg. 2019;107:941-6. https://doi.org/10.1016/j. athoracsur.2018.08.069.

16. Mercer RM, Wigston C, Banka R, Cardillo G, Benamore R, Nicholson AG, et al. Management of solitary fibrous tumours of the pleura: a systematic review and meta-analysis. ERJ Open Res. 2020;6:00055-2020. https://doi. org/10.1183/23120541.00055-2020. 